\title{
The autopsy debate during the COVID-19 emergency: the Italian experience
}

\author{
Anna Sapino ${ }^{1,2} \cdot$ Fabio Facchetti $^{3} \cdot$ Emanuela Bonoldi $^{4} \cdot$ Andrea Gianatti $^{5} \cdot$ Mattia Barbareschi $^{6}$ (i) $\cdot$ on behalf of $^{-}$ \\ Società Italiana di Anatomia Patologica e Citologia - SIAPEC
}

Published online: 29 April 2020

(C) Springer-Verlag GmbH Germany, part of Springer Nature 2020

February 20, 2020: the first Italian patient affected by COVID-19 is registered in Lombardy, a region in northern Italy. April 5, 2020: the Italian official health agency reports 128,948 persons positive for SARS-CoV-2 and 15,887 deaths due to COVID-19. Such severe epidemic critically impacted on the organization of the Italian National Health System. The pathology community was not excluded, and we had to face exceptional biosafety measures in both surgical pathology and post-mortem examinations.

Since the beginning of the Italian epidemic, the board of The Italian Society of Surgical Pathology and Cytology (Società Italiana di Anatomia Patologica e Citologia SIAPEC) recognized the urgency to provide national recommendations regarding post-mortem studies.

The colleagues of the National Institute for Infectious Diseases "Lazzaro Spallanzani" in Rome and of the "Luigi Sacco" Hospital in Milan contacted the Society board to give their support together with the Supreme Health Authority (Istituto Superiore di Sanità) to produce guidelines on how to perform autopsies [1]. The document states that

This article is part of the Topical Collection on Quality in Pathology

Mattia Barbareschi

mattia.barbareschi@apss.tn.it

1 Department of Medical Sciences, University of Torino, Torino, Italy

2 Candiolo Cancer Center, FPO-IRCCS, Candiolo, Torino, Italy

3 Department of Molecular and Translational Medicine, Pathology Unit, University of Brescia School of Medicine, Spedali Civili Brescia, Italy

4 Unit of Surgical Pathology and Cytogenetics, Niguarda Cancer Center, Milan, Italy

5 Unit of Pathology, Papa Giovanni XXIII Hospital, Bergamo, Italy

6 Unit of Surgical Pathology, S. Chiara Hospital, Azienda Provinciale per i Servizi Sanitari, Trento, Italy "in patients dying with SARS-CoV-2 infection, the autopsies can confirm laboratory and radiological findings and can contribute to an accurate diagnosis and to a better understanding of mechanisms of the disease." In the meantime, the SIAPC Board accepted to collaborate with the Scientific Society of Hospital Forensic Medicine of the National Health System (COMLAS) to produce a joint document, which was available on the SIAPEC web site on March 22 [2]. The document was translated in English and published on Pathologica, the official SIAPEC journal [3]. We acknowledged that safety of operators must be the main goal to confront with, and we defined the criteria to perform the post-mortem studies in suspect, probable, or confirmed COVID-19 cases [2,3]. In this document, we highlighted that post-mortem examination does not have a primary diagnostic role: the diagnosis relies on the clinical picture, the results of laboratory tests (nasal and oropharyngeal swabs aimed at identifying viral RNA with PCR techniques), and typical lung CT scans. Autopsy may still have a clinical role in selected cases, but should more appropriately be restricted to research projects, where the collection of tissues may shed new insights on the disease-induced organ damages. We also suggested that tissue sampling on corpses can be done using core biopsies, as suggested by one of the first reports of an autopsy case of COVID-19 [4].

Apart from suspect, probable, or confirmed COVID-19 cases, autopsies might be requested for patients not suspected to be SARS-Cov2 positive. Due to the high level of contagion in hospitals and in the general population, any corpse is regarded as potential source of infection. Therefore, for any autopsy, we recommend always to adopt the same biosafety measures as for confirmed COVID-19 cases. In addition, in cases of autopsies without apparent SARS-CoV-2 infection, we recommend (i) to discuss with the clinicians the reason why the post-mortem examination is requested; (ii) and if available, to perform nasal-oropharyngeal swabs on corpses 
within $2 \mathrm{~h}$ of death to assess the presence of SARS-CoV-2 infection to implement the safety measures [3].

In the meantime, pathologists from Bergamo's Giovanni XIII hospital, a city in Lombardy with very high incidence of SARS-CoV-2 infection causing more than 1000 deaths, have performed autopsies on selected cases (e.g., young persons or subjects without any co-morbidity, or with atypical therapeutic response), adopting a minimally invasive protocol, i.e., they did not perform complete evisceration (in particular, no brain and intestine removal) (Gianatti et al., manuscript in preparation). These data will be of great interest for our community.

On April 1, the Italian Ministry of Health published an official document on autopsies during the SARS-CoV2 epidemics [5]. Below is a summary of the main indications:

- For the entire period of the emergency phase, autopsies or post-mortem diagnostic studies should not be performed in full-blown cases of COVID-19.

- The Judicial Authority will evaluate the possibility of limiting the assessment to the sole external inspection of the corpse, in all cases where an autopsy is not strictly necessary for forensic reasons.

- The Health Departments of each region will give the criteria to limit the execution of the autopsies to those aimed at diagnosing the cause of death, strictly limiting those for study purposes.

- A careful preventive assessment of the risks and benefits associated with any autopsy request should be done.

- Autopsies and any post-mortem diagnostic activity can only be carried out in sector rooms that guarantee adequate safety conditions and personnel must wear personal protection equipment, including respiratory (FFP2 or higher) and protection devices for eyes and facial mucous membranes (visor or face shield) and hands (cut-resistant gloves interposed between a double pair of autopsy gloves).

- Any procedure which can produce aerosols should be avoided.

- Samples of tissues and organs for histological examinations must be immediately fixed.

There are several other documents of international regulatory agencies such as the World Health Organization (WHO) [6], Centers for Disease Control and Prevention (CDC) [7], European Centre for Disease Prevention and Control (ECDC) [8], and The Royal College of Pathologists [9, 10] which provide autopsy recommendations, all of which emphasize the importance of high level of biosafety of our activity.

In summary, while routine diagnostic activities of surgical pathology are our priority because patients with cancer and many other serious diseases still need our diagnoses, our main obligation is to safeguard the health of pathology staff (technicians, biologists, pathologists, administrative) [10-12]. Autopsies should be restricted to well-motivated cases and performed in accordance with strict biosafety rules. Moreover, as post-mortem histopathological findings could play a role in understanding the pathophysiology of the SARS-CoV-2 infection, SIAPEC is promoting a national study group to deal with diagnostic and organizational challenges related to the ongoing emergency and is willing to create a repository of micro- and macroscopic images illustrating the organ damage induced by SARS-CoV-2. A repository of these images will certainly be of help in the diagnostic activity and could be an important source for future collaborative studies.

Authors' contributions All authors contributed to the discussion of the manuscript.

\section{Compliance with ethical standards}

Conflict of interest The authors declare that they have no conflict of interest.

Ethical approval Not applicable.

\section{References}

1. Rapporto ISS COVID-19 n. 6/2020 - Procedura per l'esecuzione di riscontri diagnostici in pazienti deceduti con infezione da SARSCoV-2. Versione del 23 marzo 2020. https:/www.iss.it/documents/ 20126/0/Rapporto+COVID-19+n.+6_2020+Autopsie+v27+ marzo.pdf/c4b363a1-a246-c36c-d007-ae24ed7e648b?t= 1585307031219. Accessed 8 April 2020

2. Infezione respiratoria da COVID-19 DOCUMENTO SU AUTOPSIA E RISCONTRO DIAGNOSTICO"prodotto da COMLAS e SIAPEC-IAP. https://www.siapec.it/public/uploads/ archiviodocumenti/PRD\%20COVID-19-9\%20rev001\% 20010420.pdf. Accessed 8 April 2020

3. Fineschi V et al (2020) Management of the corpse with suspect, probable or confirmed COVID-19 respiratory infection - Italian interim recommendations for personnel potentially exposed to material from corpses, including body fluids, in morgue structures and during autopsy practice. Pathologica. https://doi.org/10.32074/ 1591-951X-13-20

4. Xu Z, Shi L, Wang Y, Zhang J, Huang L, Zhang C, Liu S, Zhao P, Liu H, Zhu L, Tai Y, Bai C, Gao T, Song J, Xia P, Dong J, Zhao J, Wang FS (2020) Pathological findings of COVID-19 associated with acute respiratory distress syndrome. Lancet Respir Med. 8(4):420-422. https://doi.org/10.1016/S2213-2600(20)30076-X. Erratum in: Lancet Respir Med. 2020 Feb 25

5. Indicazioni emergenziali connesse ad epidemia COVID-19 riguardanti il settore funebre, cimiteriale e di cremazione. Ministero della Salute-Direzione Generale Della Prevenzione Sanitaria. Ufficio 4 - 0011285-01/04/2020-DGPRE-DGPRE-P. http://www.trovanorme.salute.gov.it/norme/renderNormsanPdf? anno $=2020 \&$ codLeg $=73789 \&$ parte $=1 \% 20 \&$ serie $=$ null. Accessed 8 April 2020

6. WHO (2020) Infection prevention and control for the safe management of a dead body in the context of COVID-19 (2020). https:// 
apps.who.int/iris/bitstream/handle/10665/331538/WHO-COVID19-1PC_DBMgmt-2020.1-eng.pdf. Accessed 8 April 2020

7. Centers for Disease Control and Prevention. Collection and submission of postmortem specimens from deceased persons with known or suspected COVID-19, March 2020 (Interim Guidance). https:// www.cdc.gov/coronavirus/2019-ncov/hcp/guidance-postmortemspecimens.html. Accessed 8 April 2020

8. European Centre for Disease Prevention and Control. Considerations related to the safe handling of bodies of deceased persons with suspected or confirmed COVID-19. https://www.ecdc.europa.eu/en/ publications-data/considerations-related-safe-handling-bodiesdeceased-persons-suspected-or. Accessed 8 April 2020

9. The Royal College of Pathologists. Autopsy practice relating to possible cases of COVID-19 (2019-nCov, novel coronavirus from China 2019/2020). https://www.rcpath.org/uploads/assets/ d5e28baf-5789-4b0f-acecfe370eee6223/fe8fa85a-f004-4a0c-
81ee4b2b9cd12cbf/Briefing-on-COVID-19-autopsy-Feb-2020. pdf. Accessed 8 April 2020

10. Firth J (2020) Covid-19 current advice for pathologists. Pathologica. https://doi.org/10.32074/1591-951X-12-20

11. Barbareschi M, Facchetti F, Fraggetta F, Sapino A (2020) What are the priorities of pathologists' activities during COVID-19 emergency? Pathologica. https://doi.org/10.32074/1591-951X-15-20

12. Barbareschi $\mathrm{M}$ et al (2020) Biosafety in surgical pathology in the era of SARS-Cov2 pandemia. A statement of the Italian Society of Surgical Pathology and Cytology. Pathologica. https://doi.org/10. 32074/1591-951X-14-20

Publisher's note Springer Nature remains neutral with regard to jurisdictional claims in published maps and institutional affiliations. 